\title{
Integration of AM process in design cycle of metallic parts: Application to space components
}

Frédéric Duboeuf, Etienne Lemaire, Alain Remouchamps, Tom Van Eekelen, Charles Chary, Marc François, Alexandru Vargalui and Gonçalo Rodrigues

\author{
Frédéric Duboeuf. Samtech s.a. (Siemens Digital Industries Software) Belgium \\ Corresponding author: Duboeuf Frédéric. E-mail address: frederic.duboeuf@siemens.com \\ Etienne Lemaire. Samtech s.a. (Siemens Digital Industries Software) Belgium \\ Alain Remouchamps. Samtech s.a. (Siemens Digital Industries Software) Belgium \\ Tom Van Eekelen. Samtech s.a. (Siemens Digital Industries Software) Belgium \\ Charles Chary. Sonaca, Belgium \\ Marc François. Sonaca, Belgium \\ Alexandru Vargalui. ESA/ESTEC (European Space Agency), The Netherlands \\ Gonçalo Rodrigues. ESA/ESTEC (European Space Agency), The Netherlands
}

\begin{abstract}
Identified in the European strategy as a key enabling technology, Additive Manufacturing (AM) has a great potential for industries to reshape, improve and optimize product life cycle, with reduced environmental footprint such as material waste in production. Allowing to meet structural and multi-disciplinary requirements with complex freeform design at a much lower weight than high constrained conventional manufacturing, AM can benefit to numerous space applications. Beside manufacturing process development, software and process control are becoming absolutely necessary to support digitalization of industrial workflow. Dedicated tools such as Computer Aided Design (CAD), Computer Aided Engineering (CAE) and Computer Aided Manufacturing (CAM) were introduced in the digital manufacturing chain; however, their development was driven by standard manufacturing processes. Therefore, appropriate design methods for AM must emerge in a fully integrated end-to-end solution to foster and support the growth and competitiveness of AM. In order to support industrialization of AM, the European Space Agency has selected the Design4AM project, based on a strong partnership between Siemens and Sonaca, for "Development of Design Methods for AM including CAD Design, Optimization, FEM Analysis and Manufacturing features". On one hand, the project aims at combining within a comprehensive end-to-end process, topology optimization, seamless CAD data flows and predictive process simulation in the Siemens' $\mathrm{NX}^{\mathrm{TM}}$ and Simcenter ${ }^{\mathrm{TM}}$ environments. On the other hand, the integration of dedicated industrial design workflow within the enhanced Siemens Digital Innovation Platform is validated on a relevant ESA space application provided by Sonaca.
\end{abstract}

Keywords. Generative Design And Simulation, Additive Manufacturing, End-to-end Solution

\section{Introduction}

Legacy software and process control involved in Product Lifecycle Management (PLM) and initiated, for instance, as dissociated CAD/CAE/CAM tools in response to digitalization needs for conventional manufacturing, are facing new challenges this last decade. Additive Manufacturing (AM) has been subject to a closer industrial interest and emerges as being a key of the technological and economic developments. But, to leverage the full potential of these disruptive technologies and transform the entire value chain, it requires a comprehensive end-to-end integrated software system with seamless digital thread.

This can be achieved by connecting - as digital twins - products, production and performance in a closed-loop. From design process to 3D printing of metallic parts, the integrated software tools must give access to innovative concepts ready for print. To this end, a specific care must be given to provide seamless data flows within this digital chain, allowing efficient engineering loops and streamlined workflow. Furthermore, some constraints must be considered as 
Integration of AM process in design cycle of metallic parts: Application to space compo...

soon as possible in the design process:

- performance requirements: with optimized parts subject to their full environment(s), in which several configurations may be involved in service;

- manufacturing requirements: with, for instance, self-supporting structure with respect to build orientation directly generated by topology optimization, and predictive AM process simulation allowing compensation of thermal distortions.

In this context, the European Space Agency (ESA) selected Siemens and Sonaca for development of design for additive manufacturing applications. The resulting partnership is collaborating in the framework of the ongoing Design4AM project to enhance the Siemens Digital Innovation Platform for industrialization of additive manufacturing in aerospace industry.

Based on the Siemens' software solution, development of design methods dedicated for AM is progressively consolidated on relevant cases studies, before being validated on an ESA space application (supports of satellite instrument) provided by Sonaca. This will provide a robust design, sizing and AM process preparation methodology supported by a single software environment.

\section{Development of the Digital Twins for performance requirements}

In order to support flexible design cycle with generative tool for highly complex industrial applications, a generic framework has been developed to handle performance requirements within topology optimization.

First, the high accuracy requested for space applications imposes to consider not only the design space within its (limited) surrounding environment, but its full environment. The resulting model may contain an extensive number of elements not directly related to the optimized part. Topology optimization being an iterative process, this leads to substantive additional computational cost at each iteration. Furthermore, this can impact pre- and post-processing of the entire model, which are potentially more prone to errors. To address this issue, an efficient approach has been implemented within Topol, the topology optimization tool used for research conducted in the Design4AM project and available as module of Simcenter Samcef. Super-elements are introduced within the topology optimization to decouple models between the optimized part and its environment. This approach benefits also to preprocessing, by simplifying the model translation workflow between Nastran and Samcef, reducing compatibility issues with a direct reuse of Nastran super-element. This approach is illustrated in Fig. 1 on a Launcher Interface Ring of a satellite.
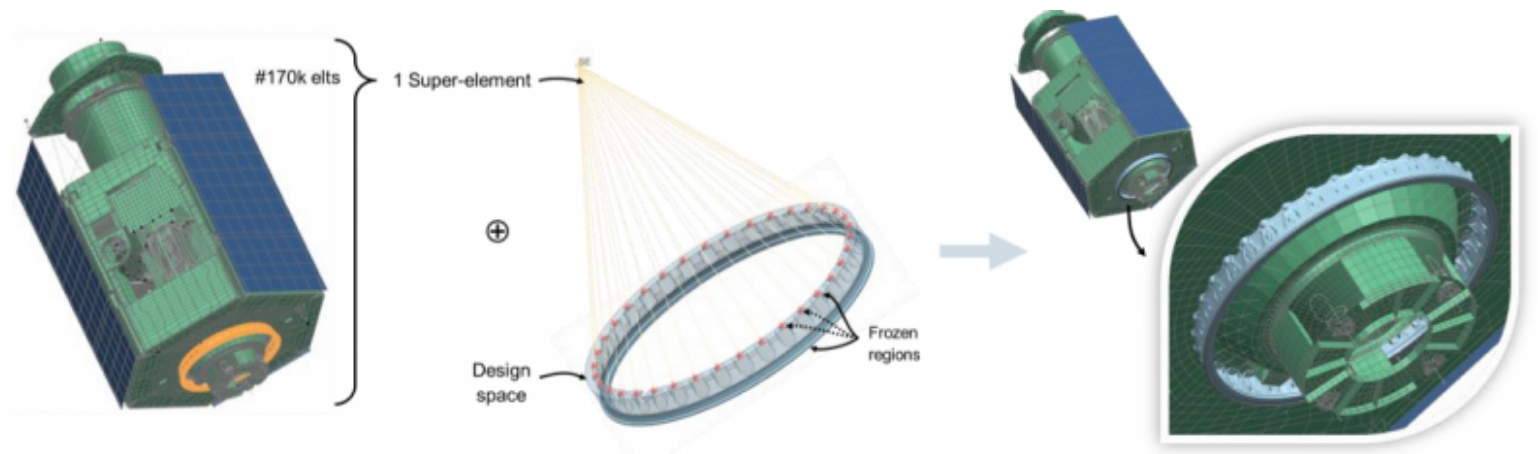

Fig. 1. Typical workflow using super-element for topology optimization of a Launcher Interface Ring of a satellite proposed by Sonaca. 
But single model-based optimization is limited. Problems involving more than one environment to consider is a common thing in space applications, where sizing must handle extreme temperatures, extreme conditions during the launch phase, locked and released configurations. By splitting the optimization into separate models, a fastidious and sensitive rework is needed to merge the different results. Therefore, a new capability to manage simultaneously several configurations of the same part to optimized (different environments potentially represented by distinct superelements, different analyses and configurations of the design space) has been implemented and allows multi-model topological optimization.

The proposed approach takes advantage of the assembly-FEM capabilities and Convergent Modeling ${ }^{\mathrm{TM}}$ technology available in Siemens' $\mathrm{NX}^{\mathrm{TM}}$ and Simcenter ${ }^{\mathrm{TM}} 3 \mathrm{D}$ environments, and leads to fully associative models diligently managing super-element connection, geometry changes and updates. This allows versatile modeling, switching quickly between multiple models, with component to optimize replaced with different models, e.g. the reference design, several proposals of design space or solutions (see Fig. 2).
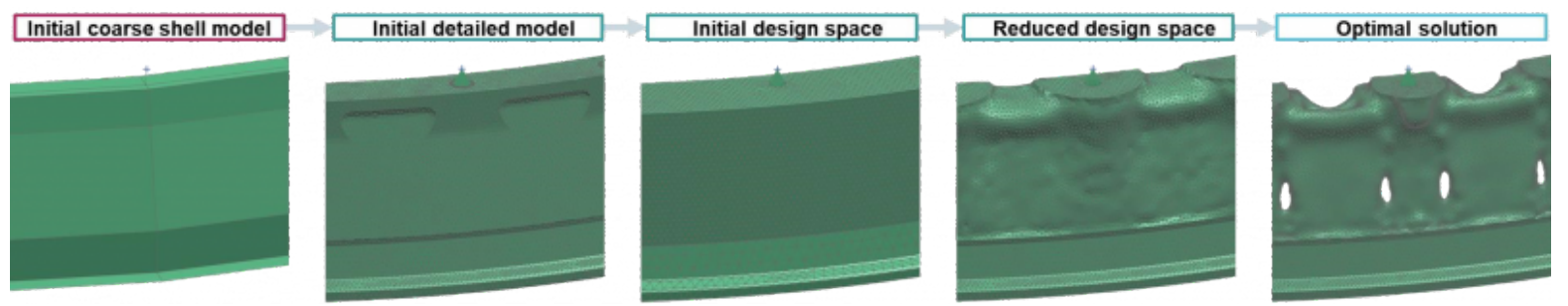

Fig. 2. Multiple models and optimal solution of a Launcher Interface Ring of a satellite proposed by Sonaca and supported by associative modeling.

\section{Development of the Digital Twins for manufacturing requirements}

Integration of manufacturing constraints has progressively been considered into the design process. Starting from trial and error design procedures, design rules have emerged and been included at the CAD level. Initially neglected during the optimization process, some manufacturing constraints can be formulated and embedded in topology optimization. Due to the layer-by-layer process of AM, specific care must be given to prevent sagging and warping of 3D printed parts. Such issues can happen, respectively, during the initial build and as the print progresses, on overhanging features. Therefore, past a certain angle support structures must be introduced. Because of the non-negligible impact on pre- and post-processing of such non-design features, developing topology optimization considering self-supported results is of interest. To this end, the problem is formulated [1] as an overhang constraint allowing to control the overhanging angle of the structural members during the optimization process, for a given build orientation (see Fig. 3). Performances of the self-supported design may differ depending on the orientation of the part to print on the build tray. Therefore, investigation will be performed to select the best orientation to print. 

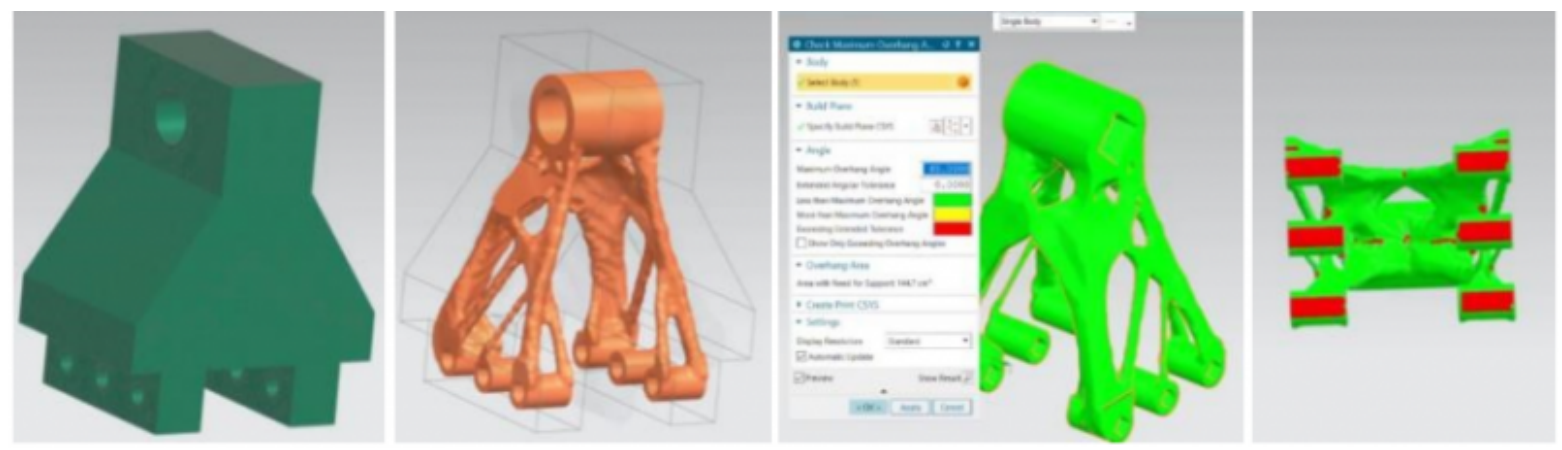

Fig. 3. Topological optimization of an additive manufactured support bracket under $45^{\circ}$ overhang constraint.

In order to provide a predictive digital twin of the production, AM process simulation has been introduced in the digital chain. The Powder Bed Fusion (PBF) printing process involves several thermal states impacting the resulting metallic part that must be considered. Indeed, a printed part results from the fusion of the powder in a layer-by-layer process, followed by its cooling. This process can be described by three temperatures (the solidus, the reference and the environment), dissociating two domains for the physics of deformation. While the standard thermal shrinkage coefficient is used for the cooldown after printing, a dedicated shrinkage models the deformation between solidus during the PBF process.

The overall precision of the simulation of the PBF process results in the accuracy of the model to represent distortion and part shrinkage from residual stress formed during the additive process. Therefore, effective layer shrinkage parameter must be a function of the stiffness. To define this constitutive material law, a specific calibration procedure has been developed (see Fig. 4), combining experimental measures and simulations results obtained on calibration parts.
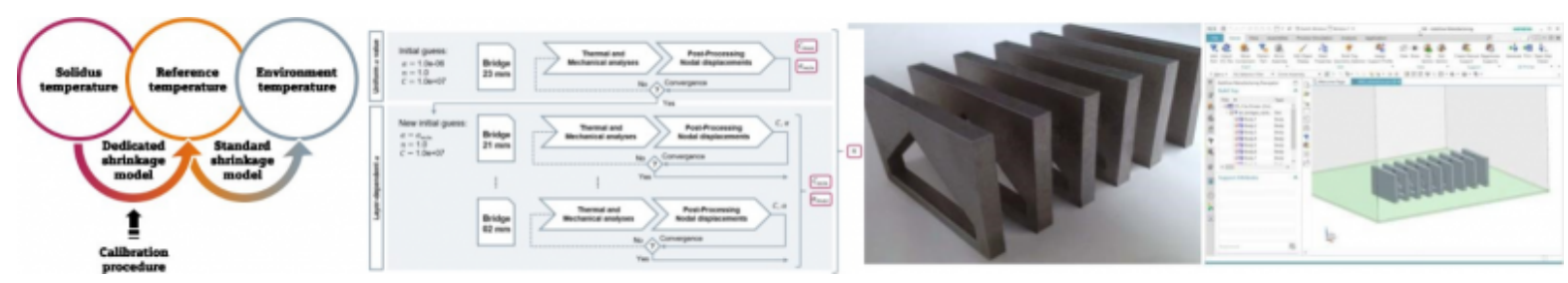

Fig. 4. Calibration procedure for AM process simulation of metallic parts. From left to right: shrinkage model fed by the detailed calibration procedure, combining experimental and simulation results.

Once the calibration results obtained for a print job, i.e. a couple of material and process parameters, the AM process simulation can be performed. Build preparation and run of the simulation on the previously optimized bracket design are illustrated in Fig. 5. 

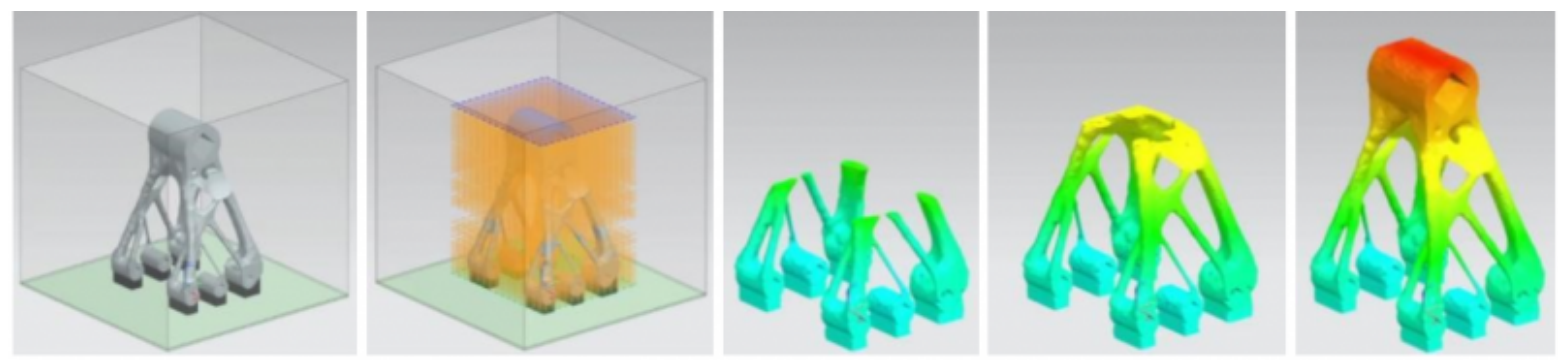

Fig. 5. Additive manufacturing process simulation of topology optimized support bracket.

\section{Validation on the breadboard of the Design4AM project}

A relevant ESA space application provided by Sonaca has been selected as breadboard of the Design4AM project for the validation of the proposed approach developed within the Siemens Digital Innovation Platform and dedicated to design for AM.

The MicroWave Imager (MWI), illustrated in Fig. 6, is part of the payload complement of a series of three MetOp Second Generation (MetOp-SG) Satellite B to be launch from 2024 to 2038, continuing and enhancing meteorological observation from polar orbit, as part of the next generation EUMETSAT Polar System (EPS-SG). The instrument is a total-power microwave radiometer, scanning conically the earth to provide calibrated and geolocated measurements, in order to monitor precipitation and sea ice extent. Such information will serve operational meteorology, oceanography, sea-ice/snow/land surface observation and climate applications.
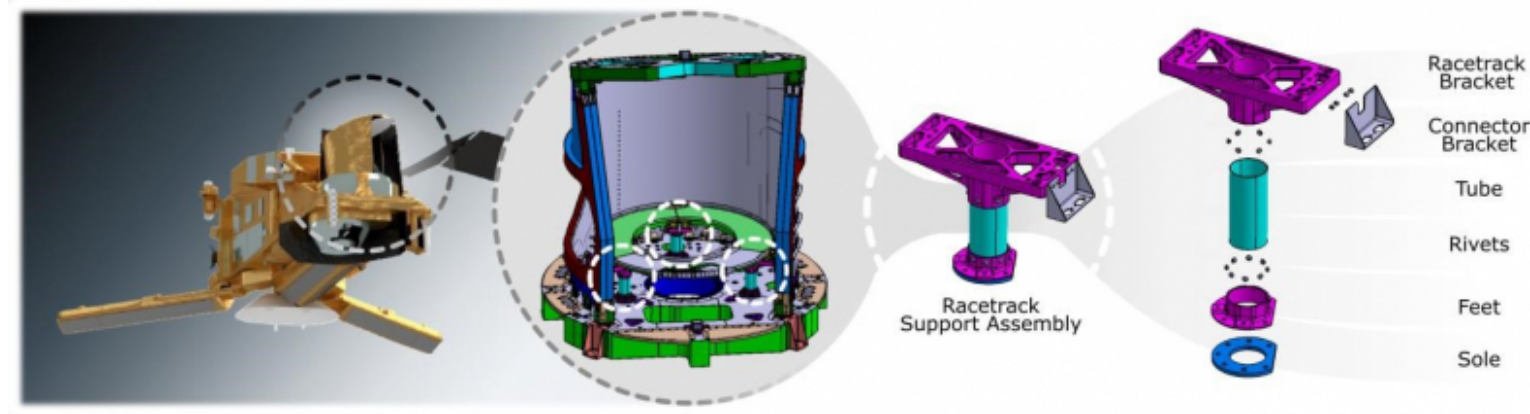

Fig. 6. Breadboard of the Design4AM project, form left to right: illustration of the MetOp-SG Satellite B (image credit: Airbus DS); one of its payloads the MWI instrument; the initial design of the racetrack support assembly and its components.

In service, the scan mechanism is traveling along the racetrack for calibration of the instrument purpose. Therefore, sizing of the three racetrack supports is critical for the performance of the instrument. Furthermore, the initial design solution for the racetrack support requires assembly with multiple components produced by conventional manufacturing process.

Therefore, by investigating AM process for an innovative design of this application, and combining the enhanced digital chain with the integrated workflow, substantial gains with regard to performance, production, assembly lead time and reduction of the environmental footprint (actually 93\% of material is lost in conventional machining corresponding to 
Integration of AM process in design cycle of metallic parts: Application to space compo...

$23.1 \mathrm{~kg}$ of material waste per instrument with three supports) are expected.

\section{Acknowledgements}

This work was supported by the European Space Agency (ESA) and the Belgian Federal Science Policy Office (BELSPO) under the Design4AM contract No. 4000125583/18/NL/BJ as part of ESA's General Support Technology Programme (GSTP). The view expressed herein can in no way be taken to reflect the official opinion of the European Space Agency.

Illustration of the Launcher Interface Ring designed for Additive Manufacturing has been obtained within the framework of the LIRAM activity (G61A-036QT) carried out by Sonaca with CRM under the GSTP program of and funded by the European Space Agency. The views expressed herein can in no way be taken to reflect the official opinion of the European Space Agency.

\section{Bibliography}

[1] M. Langelaar, Topology optimization of 3D self-supporting structures for additive manufacturing. Additive Manufacturing, 2016, 12, 60-70.

PDF automatically generated on 2021-05-20 06:22:40

Article url: https://popups.uliege.be/esaform21/index.php?id=4172

published by ULiège Library in Open Access under the terms and conditions of the CC-BY License

(https://creativecommons.org/licenses/by/4.0) 